\title{
Case-Case-Control Study of Risk Factors for Nasopharyngeal Colonization with Methicillin-Resistant Staphylococcus aureus in a Medical-Surgical Intensive Care Unit
}

\author{
Edson Carvalho de Melo ${ }^{1,2}$ and Carlos Magno Castelo Branco Fortaleza ${ }^{1,2}$ \\ ${ }^{1}$ Departamento de Doenças Tropicais e Diagnóstico por Imagem, Faculdade de Medicina de Botucatu, UNESP - Universidade Estadual Paulista; \\ ${ }^{2}$ Hospital Estadual Bauru, Faculdade de Medicina de Botucatu, UNESP - Universidade Estadual Paulista; Botucatu, SP, Brazil
}

\begin{abstract}
Nasopharyngeal colonization with methicillin-resistant Staphylococcus aureus (MRSA) often precedes the development of nosocomial infections. In order to identify risk factors for MRSA colonization, we conducted a casecase-control study, enrolling 122 patients admitted to a medical-surgical intensive care unit (ICU). All patients had been screened for nasopharyngeal colonization with $S$. aureus upon admission and weekly thereafter. Two casecontrol studies were performed, using as cases patients who acquired colonization with MRSA and methicillinsusceptible $S$. aureus (MSSA), respectively. For both studies, patients in whom colonization was not detected during ICU stay were selected as control subjects. Several potential risk factors were assessed in univariate and multivariable (logistic regression) analysis. MRSA and MSSA were recovered from nasopharyngeal samples from 27 and 10 patients, respectively. Independent risk factors for MRSA colonization were: length-of-stay in the ICU (Odds Ratio $[O R]=1.12,95 \%$ Confidence Interval $[C I]=1.06-1.19, p<0.001)$ and use of ciprofloxacin $(O R=5.05,95 \% C I=1.38-21.90$,

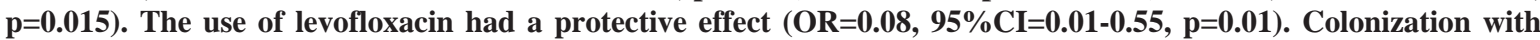
MSSA was positively associated with central nervous system disease $(O R=7.45,95 \% C I=1.33-41.74, p=0.02)$ and negatively associated with age $(\mathrm{OR}=0.94,95 \% \mathrm{CI}=0.90-0.99, \mathrm{p}=0.01)$. In conclusion, our study suggests a role for both cross-transmission and selective pressure of antimicrobials in the spread of MRSA.
\end{abstract}

Key-Words: Methicillin-resistant Staphylococcus aureus, nasopharyngeal colonization, intensive care unit.

The latter decades witnessed a substantial increase in the incidence of hospital-acquired methicillin-resistant Staphylococcus aureus (MRSA) infections [1]. This phenomenon is particularly worrisome in Intensive Care Units (ICU). Recent reports state that more than half $S$. aureus strains from ICU-acquired infections are MRSA [2]. This picture has a significant impact on patients mortality and hospital costs [3,4].

Nasopharyngeal colonization with MRSA often precedes the development of infection [5]. Also, colonized patients may transmit MRSA to other individuals, thus contributing to the spread of this pathogen all through the hospital. For this reason, infection control guidelines have recommended the active search of colonized patients, followed by prompt institution of isolation precautions [6,7].

The identification of risk factors for nasopharyngeal colonization may help in the development of strategies to prevent MRSA spread in healthcare settings. These factors have often been addressed in observational epidemiologic studies.

Methodological designs of epidemiological studies that aim to identify risk factors for the acquisition of multidrugresistant pathogens (such as MRSA) have been a matter of intense debate. In a systematic review, Harris et al. [8] observed

Received on 17 July 2009; revised 10 November 2009.

Address for correspondence: Dr. Carlos Magno Castelo Branco Fortaleza. Departamento de Doenças Tropicais e Diagnóstico por Imagem, Faculdade de Medicina de Botucatu. Distrito de Rubião Júnior, Botucatu, São Paulo State, Brazil - Zip code: 18618-970. Email: cmfortaleza@uol.com.br. Phone: 551438116212 - FAX: 55143815 9898.

The Brazilian Journal of Infectious Diseases

2009;13(6):398-402. (C) 2009 by The Brazilian Journal of Infectious Diseases and Contexto Publishing. All rights reserved. that most studies on this subject had their validity limited for one of the following reasons: (a) incorrect selection of the control group; (b) no adjustment for severity-of-illness; and (c) no adjustment for time of exposure to the risk.

The primary objective of our study was to identify risk factors for MRSA nasopharyngeal colonization in a medicalsurgical ICU where this pathogen maintains hyperendemic levels. The study design also allowed us to identify predictors of colonization with methicillin-susceptible S. aureus (MSSA) strains. In order to avoid biases, we strictly followed current methodological recommendations, with special attention to the three issues cited in the previous paragraph.

The study was conducted in the medical-surgical ICU from Hospital Estadual Bauru (HEB), one of the teaching hospitals from Faculdade de Medicina de Botucatu. HEB has 285 active beds and serves an area of approximately one million inhabitants. The hospital has a microbiology laboratory and an active Infection Control Committee (ICC).

Since 2005, the ICC collects surveillance cultures from patients admitted to the medical-surgical ICU in order to detect MRSA colonization. Cultures are collected upon admission and weekly thereafter. Briefly, nasopharyngeal secretions are collected with swabs and transported in Stuart media. Latter they are inoculated in mannitol salt agar and blood agar plates. The identification of $S$. aureus is performed through coagulase test. Resistance to methicillin is detected in disk diffusion tests (using oxacillin and cefoxitin disks) according to criteria from the Clinical and Laboratory Standards Institute [9].

Our study had a retrospective, case-case-control design, as described by Kaye et al. [10]. In this design, two casecontrol studies are performed. In the first, patients carrying drug-resistant strains (e.g., MRSA) are selected as cases. In the other, case patients are those individual that carry drugsusceptible strains (e.g., MSSA). For both studies, control 
subjects are selected among individuals from the source population, which do not harbor neither susceptible nor resistant strains. The rationale of this design is the possibility of simultaneously identifying risk factors for acquisition of the organism as a whole and for resistant strains in particular. We retrospectively analyzed results from surveillance cultures performed from March 2005 through May 2006. Patients were included in our study if they had been admitted to the ICU for at least 48 hours during the study period and had surveillance cultures (nasopharyngeal swabs) collected during their stay. Exclusion criteria were: a stay of less that 48 hours; no surveillance culture collected; positive cultures for $S$. aureus before or in the first 48 hours after admission to the ICU.

For case-control study \#1, case patients were defined as individuals who had at least one MRSA-positive surveillance culture while staying in the ICU. For case-control study \#2, case patients' definition included the presence of at least one MSSA positive surveillance culture during ICU stay. For both studies, the control group was formed by all individuals from the study period in which $S$. aureus was not recovered.

Patient data were recovered from medical charts and laboratory files. Underlying conditions were defined following the guidelines in International Classification of Diseases [11]. Severity of illness was assessed using the Acute Physiology and Chronic Health Evaluation (APACHE) II score [12]. Hospital admissions in the previous year and transfer from other hospitals were also recorded. All other data were analyzed from the day of admission to our hospital up to the isolation of $S$. aureus for case patients and up to the last negative culture for control subjects. Data included performance of surgery or other invasive procedure; use of steroids or other immune-suppressing drugs and use of antimicrobials. Time at risk was assessed using two different variables: "time in the hospital" (defined as time from admission to the hospital up to the isolation of $S$. aureus for case patients and up to the last negative culture for control subjects) and "time in the ICU” (defined in a similar fashion, but counted from the day of admission to the ICU).

Data were analyzed with epidemiological softwares: EPI INFO for Windows, version 3.2 (Centers for Disease Control and Prevention) and SPSS version 15.0 (SPSS inc). Each variable was submitted to univariate analysis. Fisher's exact test (for binomial variables) and Student's t test or the MannWhitney test (for numeric variables) were used. For multivariable analysis, we used a stepwise forward selection process [13]. Variables were gradually inserted in the model according to their effect. A p value of 0.05 was set as a limit for inclusion or removal from the model. It was also set as the final limit for statistical significance.

Of note, this project was fully approved by the reference Reseach Ethics Committee (Instituto Lauro de Souza Lima, City of Bauru, Brazil).

A total number of 122 patients met the inclusion criteria for our study. MRSA and MSSA strains were recovered from nasopharyngeal swabs from 27 and 10 patients, respectively.
In case-control \#1 (Table 1), only "time in the ICU” (Odds Ratio[OR]=1.12, 95\% Confidence Interval[CI]=1.06-1.19, $\mathrm{p}<0.001)$ and previous use of ciprofloxacin ( $\mathrm{OR}=5.05$, $95 \% \mathrm{CI}=1.38-21.90, \mathrm{p}=0.015$ ) were independent risk factors for MRSA nasopharyngeal colonization. On the other hand, previous use of levofloxacin had a protective effect $(\mathrm{OR}=0.08$, 95\%CI=0.01-0.55, $\mathrm{p}=0.01$ ).

Results from case-control \#2 are presented in Table 2. We found that MSSA colonization was positively associated with Central Nervous System disease $(\mathrm{OR}=7.45$, 95\%CI=1.33-41.74, $\mathrm{p}=0.02)$ and negatively associated with age (OR=0.94, 95\%CI=0.90-0.99, $\mathrm{p}=0.01$ ).

Many studies address the importance of crosstransmission and/or selective pressure of antimicrobials as major contributors to the emergence and spread of multidrugresistant bacteria. Determining which one of these factors is predominant in the epidemiology of a specific pathogen is primary for designing preventive strategies [14].

In our study, length-of-stay in the ICU was a significant risk factor for MRSA colonization. This finding has been reported by others, and points out to the occurrence of crosstransmission within the unit $[15,16]$. On the other hand, our results suggest that ecological pressure of antimicrobial use may also play a role in MRSA epidemiology in our hospital. A recent study by Weber et al. identified the previous use of quinolones (ciprofloxacin and levofloxacin) as risk factors for MRSA acquisition [17]. Interestingly, evidence from experimental studies suggests that sub-inibitory concentrations of quinolones enhance $S$. aureus colonization ability, specially for MRSA strains [18].

Our findings slightly differ from those reported by Weber et al. While the use of ciprofloxacin increased the risk of MRSA colonization, levofloxacin had a protective effect. We hypothesize that the much greater activity of levofloxacin against Gram-positive pathogens may have been sufficient to inhibit MRSA colonization. Unfortunately, specimens from surveillance cultures were not routinely tested for susceptibility to quinolones in our hospital. Perhaps this information could help us elucidate this issue.

Additional risk factors for MRSA acquisition in ICUs have been identified by other authors: severity-of-illness and use of invasive (particularly intravascular) devices [19]. Even though these variables were included in our analysis, they were not significant predictors for MRSA colonization.

Case-case-control studies allow investigators to simultaneously analyze risk factors for the acquisition of resistant and susceptible bacterial strains [10]. Results from these studies can be grouped in three categories: (a) variables that are risk factors only for resistant strains; (b) variables that are risk factors only for susceptible strains; and (c) variables that are risk factors for both susceptible and resistant strains. Results in category (c) should be interpreted as risk factors for the target organism as a whole. In our study, there was no concordance among risk factors for MRSA and MSSA. Age was negatively associated with MSSA carriage. We may

www.bjid.com.br 
Table 1. Risk factors for nasopharyngeal colonization with Methicillin-resistant Staphylococcus aureus: univariate and multivariable analysis.

\begin{tabular}{|c|c|c|c|c|c|c|}
\hline \multirow[t]{2}{*}{ Risk Factors } & \multicolumn{4}{|c|}{ Univariate analysis } & \multicolumn{2}{|c|}{ Multivariable analysis } \\
\hline & Cases $(n=27)$ & Controls (n=85) & OR $(95 \% C I)$ & $\mathbf{p}$ & OR (95\%CI) & $\mathbf{p}$ \\
\hline \multicolumn{7}{|l|}{ Demographic data } \\
\hline Male sex & $17(63.0)$ & $50(58.8)$ & $1.19(-.49-2.90)$ & 0.7 & & \\
\hline Age (mean) & 61.8 & 65.1 & $\ldots$ & 0.38 & & \\
\hline \multicolumn{7}{|l|}{ Comorbidities } \\
\hline Cardiac disease & $6(22.2)$ & 15 (21.8) & $1.06(0.34-3.03)$ & 0.91 & & \\
\hline Pulmonary disease & $4(14.8)$ & $27(32.1)$ & $0.37(0.12-1.17)$ & 0.08 & & \\
\hline Renal disease & 3 (11.1) & $7(8.2)$ & $1.39(0.33-5.81)$ & 0.7 & & \\
\hline Liver disease & $4(14.8)$ & 13 (15.3) & $0.96(0.29-3.25)$ & 1.00 & & \\
\hline Diabetes mellitus & $9(33.3)$ & 28 (32.9) & $1.02(0.41-2.55)$ & 0.97 & & \\
\hline Central nervous & $6(22.2)$ & 33 (38.8) & $0.45(0.17-1.23)$ & 0.12 & & \\
\hline system disease & & & & & & \\
\hline Solid malignancy & $1(3.7)$ & $9(10.6)$ & $0.33(0.04-2.69)$ & 0.45 & & \\
\hline Aids & $2(7.4)$ & $5(5.9)$ & $1.28(0.23-7.01)$ & 0.67 & & \\
\hline Trauma & 0 & $2(2.4)$ & $\ldots$ & 1.00 & & \\
\hline APACHE II (median) & 24 & 23 & & 0.38 & & \\
\hline \multicolumn{7}{|l|}{ Data related to admission } \\
\hline $\begin{array}{l}\text { Previous admission } \\
\text { to our hospital** }\end{array}$ & $11(42.3)$ & $44(51.8)$ & $0.68(0.28-1.66)$ & 0.4 & & \\
\hline Previous admission & $5(18.5)$ & $31(36.5)$ & $0.42(0.14-1.21)$ & 0.1 & & \\
\hline $\begin{array}{l}\text { to other hospitals* } \\
\text { Time in the hospital } \\
\text { (median) }\end{array}$ & 24 & 11 & $\ldots$ & $<0.001$ & & \\
\hline $\begin{array}{l}\text { Time in the ICU } \\
\text { (median) }\end{array}$ & 19 & 8 & $\ldots$ & $<0.001$ & $1.2(1.06-1.19)$ & $<0.001$ \\
\hline \multicolumn{7}{|l|}{ Immunity } \\
\hline Neutropenia & $1(3.7)$ & 0 & & 0.24 & & \\
\hline Use of steroids & 14 (51.9) & 39 (45.9) & $1.27(0.53-3.02)$ & 0.59 & & \\
\hline Use of other immune & $1(3.7)$ & 0 & $\ldots$ & 0.24 & & \\
\hline suppressing drugs & & & & & & \\
\hline \multicolumn{7}{|c|}{ Procedures and invasive devices } \\
\hline Surgery & $9(34.6)$ & $12(14.1)$ & $3.22(1.17-8.87)$ & 0.04 & & \\
\hline Menchanical ventilation & 25 (92.6) & $68(80.0)$ & $2.12(0.67-14.51)$ & 0.15 & & \\
\hline Urinary catheter & $27(100)$ & 79 (92.9) & & 1.00 & & \\
\hline Central venous catheter & $23(85.2)$ & 63 (74.1) & $2.01(0.63-6.45)$ & 0.24 & & \\
\hline Parenteral nutrition & 3 (11.1) & $5(5.9)$ & $2.00(0.35-8.98)$ & 0.7 & & \\
\hline Nasogastric/ & 24 (88.9) & 73 (85.9) & $1.31(0.34-5.06)$ & 0.4 & & \\
\hline nasoenteral tube & & & & & & \\
\hline \multirow{2}{*}{\multicolumn{7}{|c|}{ Use of antimicrobials }} \\
\hline & & & & & & \\
\hline Oxacillin & $10(37.0)$ & $13(15.3)$ & $3.26(1.22-8.67)$ & 0.02 & & \\
\hline Ampicillin & $2(7.4)$ & $2(1.4)$ & $3.32(0.45-24.79)$ & 0.25 & & \\
\hline Ampicillin-sulbactam & 0 & $1(1.2)$ & & 1.00 & & \\
\hline Amoxicillin-clavulanate & $5(18.5)$ & $30(35.3)$ & $0.42(0.14-1.21)$ & 0.1 & & \\
\hline Piperacillin-tazobactam & $6(22.2)$ & $9(10.6)$ & $2.41(0.77-7.55)$ & 0.19 & & \\
\hline Cephazolin & 0 & $3(3.5)$ & & 0.32 & & \\
\hline Cephalotin & $1(3.7)$ & $3(3.5)$ & $1.05(0.11-10.55)$ & 1.00 & & \\
\hline Ceftriaxone & $2(7.4)$ & $5(5.9)$ & $1.28(0.23-7.01)$ & 0.67 & & \\
\hline Ceftazidime & $2(7.4)$ & $3(3.5)$ & $2.18(0.35-13.83)$ & 0.59 & & \\
\hline Cefepime & $19(70.4)$ & 30 (35.3) & $4.36(1.70-11.13)$ & 0.001 & & \\
\hline Imipenem & $6(22.2)$ & $6(7.1)$ & $3.76(1.10-12.87)$ & 0.04 & & \\
\hline Meropenem & $2(7.4)$ & $2(2.4)$ & $3.32(0.45-24.79)$ & 0.25 & & \\
\hline Ciprofloxacin & $10(37.0)$ & $8(9.4)$ & $5.67(1.95-16.47)$ & 0.002 & $5.05(1.38-21.90)$ & 0.015 \\
\hline Levofloxacin & 3 (11.1) & 28 (32.9) & $0.25(0.07-0.92)$ & 0.03 & $0.08(0.01-0.55)$ & 0.01 \\
\hline Amikacin & $1(3.7)$ & $4(4.7)$ & $0.78(0.08-7.28)$ & 1 & & \\
\hline Gentamycin & 1 (3.7) & $2(2.4)$ & 1.59 (0.14-18.32) & 0.57 & & \\
\hline Clindamycin & $6(22.2)$ & 11 (12.9) & $1.92(0.64-5.81)$ & 0.35 & & \\
\hline Metronidazole & 8 (29.6) & 16 (18.8) & $1.82(0.68-4.88)$ & 0.23 & & \\
\hline Vancomycin & 11 (40.7) & 11 (12.9) & $4.02(1.71-12.51)$ & 0.002 & & \\
\hline
\end{tabular}

Data are in number(\%), unless otherwise specified. Statistically significant results are presented in bold type. OR, Odds Ratio; CI, Confidence Internval; APACHE, Acute Physiology and Chronic Health Evaluation; ICU, Intensive Care Unit. *Admissions in the past year. 
Table 2. Risk factors for nasopharyngeal colonization with Methicillin-susceptible Staphylococcus aureus: univariate and multivariable analysis.

\begin{tabular}{|c|c|c|c|c|c|c|}
\hline \multirow[t]{2}{*}{ Risk Factors } & \multicolumn{4}{|c|}{ Univariate analysis } & \multicolumn{2}{|c|}{ Multivariable analysis } \\
\hline & Cases $(n=10)$ & Controls (n=85) & OR $(95 \% \mathrm{CI})$ & $\mathbf{p}$ & OR $(95 \% C I)$ & $\mathbf{p}$ \\
\hline \multicolumn{7}{|l|}{ Demographic data } \\
\hline Male sex & $5(50.0)$ & $50(58.8)$ & $0.70(0.188-2.60)$ & 0.74 & & \\
\hline \multirow{2}{*}{\multicolumn{7}{|c|}{ Comorbidities }} \\
\hline & & & & & & \\
\hline Cardiac disease & $3(30.0)$ & $15(21.8)$ & $1.60(0.38-6.80)$ & 0.69 & & \\
\hline Pulmonary disease & $1(10.0)$ & $27(32.1)$ & $0.24(0.03-1.95)$ & 0.27 & & \\
\hline Renal disease & 0 & $7(8.2)$ & $\ldots$ & 1.00 & & \\
\hline Liver disease & 0 & 13 (15.3) & & 0.35 & & \\
\hline Diabetes mellitus & $2(20.0)$ & $28(32.9)$ & $0.51(0.10-2.56)$ & 0.5 & & \\
\hline Central nervous & & & & & & \\
\hline system disease & $8(80.0)$ & 33 (38.8) & $6.30(1.26-31.52)$ & 0.02 & 7.45 (1.33-41.74) & 0.02 \\
\hline Solid malignancy & 0 & 9 (10.6) & & 0.59 & & \\
\hline Aids & $2(20.0)$ & $5(5.9)$ & $4.00(0.67-24.05)$ & 0.16 & & \\
\hline Trauma & 0 & $2(2.4)$ & ... & 1.00 & & \\
\hline APACHE II (median) & 17.5 & 23 & $\ldots$ & 0.047 & & \\
\hline \multicolumn{7}{|c|}{ Data related to admission } \\
\hline $\begin{array}{l}\text { Previous admission } \\
\text { to our hospital* }\end{array}$ & $3(30.0)$ & 44 (51.8) & $0.40(0.10-1.65)$ & 0.32 & & \\
\hline Previous admission & $1(10.0)$ & 31 (36.5) & $0.19(0.02-1.60)$ & 0.16 & & \\
\hline Time in the hospital & 11 & 11 & $\ldots$ & 0.82 & & \\
\hline $\begin{array}{l}\text { Time in the ICU } \\
\text { (median) }\end{array}$ & 9.5 & 8 & $\ldots$ & 0.94 & & \\
\hline \multicolumn{7}{|l|}{$\begin{array}{l}\text { (median) } \\
\text { Immunity }\end{array}$} \\
\hline Neutropenia & 0 & 0 & & & & \\
\hline Use of steroids & $3(30.0)$ & 39 (45.9) & $0.51(0.12-2.09)$ & 0.5 & & \\
\hline Use of other immune & 0 & 0 & $\ldots$ & $\ldots$ & & \\
\hline suppressing drugs & & & & & & \\
\hline \multicolumn{7}{|c|}{ Procedures and invasive devices } \\
\hline Surgery & 0 & $12(14.1)$ & & 0.35 & & \\
\hline Menchanical ventilation & $9(90.0)$ & $68(80.0)$ & $2.25(0.27-19.00)$ & 0.68 & & \\
\hline Urinary catheter & 10 (100) & 79 (92.9) & & 1.00 & & \\
\hline Central venous catheter & $5 \quad 5(50.0)$ & $63(74.1)$ & $0.35(0.09-1.32)$ & 0.14 & & \\
\hline Parenteral nutrition & 0 & $5(5.9)$ & $\ldots$ & 1.00 & & \\
\hline Nasogastric/ & & & & & & \\
\hline nasoenteral tube & $9(90.0)$ & $73(85.9)$ & $1.48(0.17-12.76)$ & 1.00 & & \\
\hline \multirow{2}{*}{\multicolumn{7}{|c|}{ Use of antimicrobials }} \\
\hline & & & & & & \\
\hline Oxacillin & 0 & $13(15.3)$ & $\ldots$ & 0.35 & & \\
\hline Ampicillin & 0 & $2(1.4)$ & $\ldots$ & 1.00 & & \\
\hline Ampicillin-sulbactam & 0 & 1 (1.2) & & 1.00 & & \\
\hline Amoxicillin-clavulanate & $2(20.0)$ & 30 (35.3) & $.46(0.00-2.30)$ & 0.49 & & \\
\hline Piperacillin-tazobactam & $1(10.0)$ & 9 (10.6) & $0.94(0.11-8.29)$ & 1.00 & & \\
\hline Cephazolin & 0 & $3(3.5)$ & $\ldots$ & 1.00 & & \\
\hline Cephalotin & 0 & 3 (3.5) & & 1.00 & & \\
\hline Ceftriaxone & $1(10.0)$ & $5(5.9)$ & $1.78(0.19-16.95)$ & 0.5 & & \\
\hline Ceftazidime & $0(10.0)$ & 3 (3.5) & & 1.00 & & \\
\hline Cefipime & $3(30.0)$ & 30 (35.3) & $0.79(0.19-3.27)$ & 1.00 & & \\
\hline Imipenem & 0 & $6(7.1)$ & ... & 1.00 & & \\
\hline Meropenem & $02(2.4)$ & .. 1.00 & & & & \\
\hline Ciprofloxacin & $1(10.0)$ & $8(9.4)$ & $1.07(0.12-9.56)$ & 1.00 & & \\
\hline Levofloxacin & $1(10.0)$ & $28(32.9)$ & $0.23(0.03-1.86)$ & 0.27 & & \\
\hline Amikacin & 0 & $4(4.7)$ & $\ldots$ & 1.00 & & \\
\hline Gentamycin & 0 & $2(2.4)$ & & 1.00 & & \\
\hline Clindamycin & $1(10.0)$ & 11 (12.9) & $0.25(0.09-6.49)$ & 1.00 & & \\
\hline Metronidazole & $1(10.0)$ & 16 (18.8) & $0.48(0.06-4.06)$ & 0.69 & & \\
\hline Vancomycin & $2(20.0)$ & $11(12.9)$ & $1.68(0.32-8.97)$ & 0.62 & & \\
\hline
\end{tabular}

Data are in number(\%), unless otherwise specified. Statistically significant results are presented in bold type. OR, Odds Ratio; CI, Confidence Internval; APACHE, Acute Physiology and Chronic Health Evaluation; ICU, Intensive Care Unit. *Admissions in the past year. 
speculate that elder patients were exposed to factors that prevented MSSA colonization and that were not assessed in our study. Still, reasons for the finding of Central Nervous System diseases as risk factor for MSSA acquisition remain obscure.

MSSA strains are more likely to be susceptible to levofloxacin than MRSA. Therefore, one would expect that levofloxacin had at least the same protective effect against MSSA acquisition as it had for MRSA. However, this effect was not identified. This was probably due to the small number of MSSA cases, which was a major limitation in our study. It is possible that the resulting lack of statistical power accounted for the finding of entirely distinct risk factors for MRSA and MSSA.

Certain aspects of MRSA epidemiology may vary from one ICU to another. We have sought to identify risk factors that are amenable to interventions for containing its spread among critical patients in our hospital. Limiting ICU stay, reinforcing the adoption of barrier precautions and restricting the use of ciprofloxacin may have an impact on the incidence of MRSA colonization - and, ultimately, on MRSA infection and mortality.

\section{References}

1. Chambers HF. The changing epidemiology of Staphylococcus aureus? Emerg Infect Dis. 2001; 7: 178-82.

2. Klevens RM, Edwards JR, Tenover FC et al. Changes in the epidemiology of methicillin-resistant Staphylococcus aureus in intensive care units in US hospitals, 1992-2003. Clin Infect Dis. 2006 42: 389-91.

3. Gastmeier P, Sohr D, Geffers C et al. Mortality risk factors with nosocomial Staphylococcus aureus infections in intensive care units: results from the German Nosocomial Infection Surveillance System (KISS). Infection. 2005; 33:50-5.

4. Kopp BJ, Nix DE, Armstrong EP. Clinical and economic analysis of methicillin-susceptible and -resistant Staphylococcus aureus infections. Ann Pharmacother. 2004; 38:1377-82.

5. Jarvis WR. The epidemiology of colonization. Infect Control Hosp Epidemiol. 1996; 17: 47-52.

6. Muto CA, Jernigan JA, Ostrowsky B et al. SHEA guideline for preventing nosocomial transmission of multidrug-resistant strains of Staphylococcus aureus and enterococcus. Infect Control Hosp Epidemiol. 2003; 24: 362-86.
7. Calfee DP, Salgado CD, Classen D et al. Strategies to prevent transmission of Methicillin-Resistant Staphylococcus aureus in acute care hospitals. Infect Control Hosp Epidemiol. 2008; 29 (suppl.): S62-80.

8. Harris AD, Karchmer TB, Carmeli Y, Samore MH. Methodological principles of case-control studies that analyzed risk factors for antibiotic resistance: a systematic review. Clin Infect Dis. 2001; 32: $1055-61$.

9. Clinical and Laboratory Standards Institute. Performance standards for antimicrobial disk susceptibility tests; approved standard, 8th ed. Pennsylvania: CLSI; 2003.

10. Kaye KS, Harris AD, Samore M, Carmeli Y. The case-case-control study design: addressing the limitations of risk factor studies for antimicrobial resistance. Infect Control Hosp Epidemiol. 2005; 26:346-51.

11. World Health Organization. International Classification of Diseases. $10^{\text {th }}$ revision, 2005 update. Geneva, Switzerland: WHO; 2005.

12. Knaus WA, Draper EA, Wagner DR, Zimmerman JE. APACHE II: a severity of disease classification system. Crit Care Med. 1985; 13: 818-29.

13. Greenland S. Modeling and variable selection in epidemiologic analysis. Am J Public Health 1989; 79: 340-9.

14. Siegel JD, Rhinehart E, Jackson $M$ et al. Management of multidrugresistant organisms in health care settings, 2006. Am J Infect Control. 2007; 35 (10 Suppl 2): S165-93.

15. Marshall C, Harrington G, Wolfe R, Fairley CK, Wesselingh S, Spelman D. Acquisition of Methicillin-resistant Staphylococcus aureus in a large intensive care unit. Infect Control Hosp Epidemiol. 2003; 24: 322-6.

16. Oztoprak N, Cevik MA, Akinci E, Korkmaz M, Erbay A, Eren SS et al. Risk factors for ICU-acquired Methicillin-resistant Staphylococcus aureus infections. Am J Infect Control. 2006; 34:1-5.

17. Weber SG, Gold HS, Hooper DC, Karchmer AW, Carmeli Y. Fluoroquinolones and the risk for Methicillin-resistant Staphylococcus aureus in hospitalized patients. Emerg Infect Dis. 2003; 9:1415-22.

18. Bisognano C, Vaudaux P, Rohner P, Lew DP, Hooper DC. Induction of fibronectin-binding proteins and increased adhesion of Quinolone-resistant Staphylococcus aureus by subinhibitory levels of Ciprofloxacin. Antimicrob Agents Chemother. 2000; 44: $1428-37$.

19. Hardy KJ, Hawkey PM, Gao F, Oppenheim BA. Methicillin resistant Staphylococcus aureus in the critically ill. Br J Anaesth. 2004; 92: 121-30. 